\title{
Expérience amoureuse la plus difficile : Qu'en disent les garçons rapportant un vécu de violence physique dans leurs relations amoureuses?
}

\section{Anne-Julie LAFRENAYE-DUGAS ${ }^{1}$, Mylène FERNET ${ }^{1}$, Martine HÉBERT ${ }^{1}$, Martin BLAIS' et Natacha GODBOUT ${ }^{1}$}

1 Département de sexologie, Université du Québec à Montréal, Montréal, Québec

Auteur de correspondance : Mylène Fernet, Ph.D. ,Université du Québec à Montréal, Département de sexologie, C.P. 8888 Succursale Centre-Ville, Montréal (Québec), H3C 3P8.

Courriel : fernet.mylene@uqam.ca.

\section{Résumé}

Objectifs: Bien que la majorité des écrits portant sur la violence physique dans les relations amoureuses (VRA physique) s'attardent à la victimisation chez les filles, les garçons sont également à risque. Cette étude vise à documenter les expériences amoureuses de garçons ayant vécu de la VRA physique.

Méthode: Dans l'Enquête Parcours Amoureux des Jeunes, 1701 garçons ont rapporté avoir eu une relation amoureuse dans la dernière année. Parmi eux, 184 ont subi au moins un épisode de VRA physique et ont répondu à une question ouverte les invitant à décrire l'expérience la plus difficile vécue dans une relation amoureuse. Une analyse qualitative a été réalisée à partir des réponses, puis a été complétée avec des analyses corrélationnelles et de comparaison de moyennes.

Résultats: Cinq catégories conceptuelles ont émergé, relatant des difficultés relationnelles $(n=39)$ ou sexuelles $(n=9)$, des ruptures amoureuses $(n=47)$, des expériences d'infidélité $(n=57)$ ou de VRA $(n=19)$. Treize participants ne rapportaient aucun événement difficile. Seulement 15 participants ont évoqué un événement de VRA, et trois de VRA physique subie. Les analyses révèlent que plus un participant rapporte une tolérance élevée envers la violence, plus il a vécu de formes différentes de VRA.

Implications: Les témoignages recueillis soulignent la présence de plusieurs sources de détresse dans leurs relations amoureuses, cumulés à la VRA physique. Ces constats soulèvent l'importance d'aider les garçons à développer leurs capacités de résilience et à reconnaître les différentes formes de VRA, et de les soutenir dans leur recherche d'aide.

Mots-clés: Violence dans les relations amoureuses; violence physique; adolescence; garçons; intervention. 


\section{Introduction}

Dans les dernières années, nous avons été témoins de l'émergence de mouvements sociaux visant la reconnaissance du vécu des victimes de violence dans les relations amoureuses (VRA). Aux États-Unis, depuis 2011, le «Project Unbreakable » a publié plusieurs dizaines de photographies de garçons et d'hommes survivants de VRA ou de violence sexuelle tenant devant eux des affiches sur lesquelles sont inscrites des paroles de leur agresseur (Project Unbreakable, s.d.). En 2017, en Angleterre, la chaîne «BBC Three » a mis en ligne une capsule vidéo d'expérimentation sociale dans laquelle une jeune femme agresse physiquement et verbalement un jeune homme en public (environ 3 millions de visionnements). Les commentaires des témoins révèlent que plusieurs expriment que le jeune homme agressé semble «faible». Au Québec, l'équipe du projet Parcours Amoureux des Jeunes (PAJ), en partenariat avec la Table de concertation en violence conjugale de Montréal, a mis sur pied en 2016 une campagne socionumérique sur les réseaux sociaux visant à sensibiliser les jeunes et leurs parents à la violence dans les relations amoureuses (https://www.facebook.com/PAJ.UQAM). Ces différentes initiatives ont permis d'accroître la sensibilisation au sujet de la VRA et de la grande diversité de victimes, parmi lesquelles on compte des garçons adolescents.

La VRA réfère à toute tentative de dominer son partenaire par des moyens physiques (pousser ou frapper), psychologiques (menacer ou dénigrer) ou sexuels (avoir recours à la force, aux drogues ou au chantage pour avoir un rapport sexuel) (Hébert et al., 2018). Bien que les écrits scientifiques actuellement disponibles s'attardent principalement à la victimisation chez les filles, les garçons sont également à risque de subir de la VRA (Foshee et al., 2013; Hébert et al., 2017). Dans un échantillon pondéré représentatif de la population adolescente étudiant au deuxième cycle du secondaire au Québec, 15 \% des jeunes en auraient subi au moins une fois, avec une prévalence de victimisation de 12,8\% chez les garçons et de 15,7\% chez les filles (Hébert et al., 2017). Les garçons et les filles semblent démontrer des vulnérabilités différentes face à la VRA physique, les filles étant plus à risque de blessures graves et les garçons rapportant un moindre accès à des ressources reconnaissant leur victimisation (Mahalik et al., 2010; Martin et al., 2012; Muñoz-Rivas et al., 2007). Certains facteurs contribueraient spécifiquement à accroître la vulnérabilité des adolescents aux situations de VRA (Kassis et al., 2013; Ruel et al., 2020; Simon et al., 2010). Un des facteurs qui permettrait aux garçons de composer avec un vécu de violence est la résilience, c'est-à-dire l'aptitude à rebondir et s'adapter à la suite d'événements de vie difficile, mais celle-ci tend à être affectée par les normes socioculturelles sur la masculinité (Grych et al., 2015; Kassis et al., 2013; Simon et al., 2010). Par exemple, tel qu'illustré dans la vidéo présentée par la chaîne «BBC Three » (2017), ces normes présentent la VRA physique commise par une fille envers un garçon comme étant un geste plus anodin que l'inverse (Ruel et al., 2020; Simon et al., 2010).

Un autre facteur qui contribue au risque de victimisation et qui entrave la résilience des garçons est le faible sentiment de compétence face à la gestion d'une situation de VRA subie (Ali et al., 2011; Van Camp et al., 2014). Les garçons se sentiraient plus confiants d'aider quelqu'un d'autre à solliciter de l'aide qu'à chercher du soutien pour euxmêmes (Van Camp et al., 2014). Bien que la recherche de soutien après un événement difficile s'avère être un facteur de protection et une source de résilience reconnue, certains garçons sont réticents à en solliciter (Grych et al., 2015; Kassis et al., 2013). Avoir évolué dans un milieu tolérant envers la violence ou encore adhérer aux stéréotypes socioculturels de masculinité serait lié à la fois à un risque accru de VRA et à des difficultés à solliciter de l'aide après un épisode de VRA (Ali et al., 2011; Mahalik et al., 2010; Martin et al., 2012; Oransky et Marecek, 2009). En somme, les données disponibles suggèrent des spécificités de genre, tant dans la façon dont les garçons expérimentent la VRA physique, que dans les stratégies qu'ils mettent en place en réponse à ces situations de violence. De même, bien que certaines études aient documenté que les hommes tendent à ne pas interpréter comme violents ou à minimiser certains gestes qu'ils subissent (Machado et al., 2016), peu de données scientifiques actuelles ont exploré la perception que les garçons adolescents ayant subi de la VRA physique ont de leur expérience amoureuse.

\section{Objectifs de recherche}

La présente étude s'intéresse précisément au vécu de garçons ayant subi de la VRA physique et à leur perception de leur pire expérience amoureuse dans une approche mixte qualitative et quantitative. D'abord, elle vise à documenter les préoccupations de garçons ayant vécu de la violence physique dans le cadre de leurs relations amoureuses, ainsi que les différentes formes de VRA et gestes de VRA physique rapportés par les participants. Elle vise à donner la parole à ces garçons en les invitant à témoigner, de leur perspective, de l'expérience qu'ils décrivent comme étant la situation la plus difficile qu'ils ont vécue dans le contexte d'une relation amoureuse. Ensuite, elle vise à enrichir la description qualitative de ces expériences par des analyses quantitatives explorant les facteurs de risque associés à la VRA (par ex. : I'adhésion à des normales sociales favorables à la violence). 


\section{Méthode}

Les données sont issues de la vaste enquête PAJ, dont l'objectif était d'explorer et de documenter au Québec le phénomène de la VRA auprès des jeunes du secondaire dans un échantillon représentatif de 8194 jeunes. Les participants ont été recrutés dans diverses écoles publiques francophones et anglophones, et privées francophones à travers le Québec. L'échantillon est proportionnellement distribué entre les élèves de secondaire III (30,5 \%), IV (38,8 \%) et $\mathrm{V}(30,7 \%)$. Ces adolescents ont rempli une série de questionnaires comportant, entre autres, une question ouverte les invitant à décrire l'expérience la plus difficile qu'ils ont eu à vivre dans un contexte amoureux. Ce projet a été approuvé par le comité d'éthique de la recherche auprès d'êtres humains de l'Université du Québec à Montréal.

\section{Participants}

Des 8194 répondants, un total de 1701 garçons ont rapporté avoir eu une relation amoureuse dans les 12 derniers mois ou être en relation au moment de l'étude, dont 243 (14.3\%) indiquant avoir subi au moins un épisode de VRA physique. Parmi ceux-ci, 184 (75,7 \%) ont offert une réponse suffisamment complète à la question ouverte pour être utilisée à des fins d'analyse de contenu.

Des 184 participants retenus, tous se sont identifiés comme hétérosexuels, mais sept rapportaient avoir déjà vécu une expérience amoureuse avec un partenaire de même sexe. Parmi ceux qui étaient en relation amoureuse au moment de l'étude, trois (1,6\%) l'étaient avec un garçon et les autres avec une fille. Pour ceux qui n'étaient pas en relation amoureuse au moment de la participation, mais qui l'ont été dans la dernière année, six (3,3\%) étaient en relation avec un garçon et les autres avec une fille.

Les participants étaient âgés de 13 à 19 ans $(M=15,7, E . T .=1,0)$, et 22,3\% étaient de niveau secondaire III $(n=41), 41,3 \%$ secondaire IV $(n=76)$, et $35,9 \%$ secondaire $\mathrm{V}(n=66)$. Ils étaient presque tous $(98,3 \%)$ inscrits au programme de formation générale. La majorité était d'origine québécoise ou canadienne $(77,0 \%)$ et habitait avec leurs deux parents sous le même toit $(52,2 \%)$. Les caractéristiques sociodémographiques de l'échantillon sont détaillées dans le Tableau 1.

Tableau 1. Caractéristiques sociodémographiques

\begin{tabular}{lc}
\hline Caractéristiques & $\%$ \\
\hline Année scolaire & \\
Secondaire III & 22,3 \\
Secondaire IV & 41,3 \\
Secondaire V & 35,9 \\
Type de scolarité & \\
Formation générale & 98,3 \\
$\quad$ Formation préparatoire au travail & 1,7 \\
Origine ethnique/culturelle des parents & \\
Québécoise ou canadienne & 77,0 \\
Latino-américaine ou antillaise & 8,8 \\
Asiatique & 3,8 \\
Européenne & 3,8 \\
Africaine subsaharienne & 2,2 \\
Africaine du nord ou moyenne orientale & 2,2 \\
Autochtone & 1,7 \\
Autre & 0,5 \\
Structure familiale & \\
Habite avec les deux parents dans la même demeure & 52,2 \\
En garde partagée & 12,0 \\
Avec leur mère & 17,9 \\
Avec leur père & 7,1 \\
Avec un membre de la famille élargie & 3,8 \\
En famille ou centre d'accueil & 2,2 \\
Avec leur partenaire amoureux & 1,6 \\
Autre & 3,2 \\
\hline
\end{tabular}




\section{Mesures}

Des adaptations francophones du Conflict in Adolescent Dating Relationships Inventory (CADRl; Wekerle et al., 2009; Wolfe et al., 2001) et du Sexual Experiences Survey (SES; Koss et al., 2007) ont été utilisées pour évaluer la VRA physique, psychologique et sexuelle (Hébert et al., 2011). La version du CADRI utilisée contient huit énoncés, dont six sont dérivés d'une version courte du CADRI (Wekerle et al., 2009), et deux de la version longue du CADRI (Wolfe et al., 2001), et le SES comporte neuf items (Koss et al., 2007). Ces deux échelles contiennent des énoncés présentant un type de comportement de VRA que le partenaire amoureux a pu commettre envers le participant (par ex. : «Te frapper ou te donner un coup de poing ou de pied »; «Te ridiculiser ou rire de toi devant les autres »; « Avoir une relation sexuelle avec toi en utilisant des arguments ou des pressions »). Les participants étaient invités à indiquer la fréquence à laquelle un comportement de VRA s'était produit dans les 12 derniers mois à l'aide d'une échelle de type Likert variant de 0 ( «jamais ») à 3 (« six fois ou plus »). Chaque forme de VRA a été dichotomisée ( 0 - « non vécue »; 1 - « vécue »), et tel que suggéré par certains auteurs afin de reconnaître l'effet cumulatif des différentes formes de violence et de souligner que même un seul événement de VRA ne doit pas être toléré (Vagi et al., 2015), un score cumulatif indiquant le nombre de formes de VRA vécues (entre 0 et 3 formes) a été calculé. L'alpha de Cronbach pour le questionnaire complet est de ,81 dans la présente étude.

Une échelle d'attitudes envers la violence physique dans les relations amoureuses, l'Acceptance of Prescribed Norms Scale (APNS; Foshee et al., 2001) permet d'apporter des informations complémentaires sur la tolérance envers la VRA par les victimes. Cette échelle est composée de six énoncés (par ex. : "Les gars méritent parfois de se faire frapper par leur blonde » ou « Les filles méritent parfois de se faire frapper par leur chum »). Les participants ont indiqué leur degré d'accord avec ces énoncés à l'aide d'une échelle de Likert allant de 0 («Fortement en désaccord») à 3 («Fortement en accord»). Le score total peut varier de 0 à 18 et un score plus élevé signifie une plus grande tolérance de la VRA. Cette échelle démontre des qualités psychométriques satisfaisantes dans les études antérieures ( $\alpha=, 73$; Ruel et al., 2020) et dans la présente étude $(a=, 72)$. À la fin du questionnaire, les participants étaient invités à répondre à la question ouverte suivante : «Écris quelques mots sur l'expérience la plus difficile que tu as vécue dans tes relations amoureuses. Écris le contexte de la situation, donne des informations sur l'autre personne impliquée, sur ce que vous avez fait, sur tes sentiments sur le moment et après ».

\section{Stratégies d'analyse}

Des analyses descriptives ont en premier lieu été réalisées afin de documenter la prévalence des différents gestes de violence vécus par les participants. Ensuite, les réponses des jeunes à la question ouverte concernant leur pire expérience amoureuse ont été analysées de façon inductive, et ont été soumises à une analyse de contenu thématique (Fourboul, 2012). Dans le cadre de ces analyses, les participants sont considérés comme étant des experts de leurs propres expériences et des interlocuteurs clés pour témoigner des expériences difficiles dans leurs relations amoureuses, dont les expériences de violence physique (Poupart, 1997). Les analyses comportent trois étapes (Braun et Clarke, 2006). Premièrement, une grille de codification mixte a été créée, en suivant une démarche itérative et réflexive. Deuxièmement, chaque réponse a été considérée, codifiée et organisée en thèmes identifiés, autant à partir des témoignages, qu'à partir des écrits théoriques. Troisièmement, la grille a été réduite, jusqu'à l'obtention de catégories conceptuelles indépendantes et mutuellement exclusives. Chacune de ces catégories conceptuelles illustre un phénomène permettant de répondre à l'objectif de recherche. Enfin, des Test tet des corrélations ont été réalisés afin d'explorer l'adhésion à des normes sociales favorables à la VRA chez les participants et de comparer l'échantillon actuel à un échantillon ne rapportant pas de VRA.

\section{Résultats}

\section{Analyses descriptives}

Parmi les 184 adolescents retenus pour l'analyse thématique, la majorité $(84,2 \%)$ rapporte avoir vécu plus d'une forme de VRA, 152 (82,6\%) ayant aussi vécu au moins un épisode de violence psychologique et 33 (17,9\%) de la violence sexuelle au cours de la dernière année. En ce qui a trait à la VRA physique qu'ils ont vécue dans les 12 mois ayant précédé l'enquête, 65,2 \% ont été giflés ou se sont fait tirer les cheveux, 43,2 \% indiquent avoir été frappés avec le pied ou le poing, et 40,7 \% ont été poussés, secoués, bousculés ou retenus de force. 


\section{Analyse de contenu thématique}

L'analyse de contenu thématique des témoignages d'adolescents indiquant avoir subi de la VRA physique a permis de dégager cinq catégories conceptuelles. Le Tableau 2 résume les catégories et sous-catégories, indique le nombre d'extraits de discours contenus dans chacune d'elles et présente des extraits de discours illustratifs pour chaque catégorie. La catégorisation a été revue par trois chercheurs (A.-J. L.-D., M. F. et M. H.) à l'aide d'une procédure de fidélisation interjuges jusqu'à l'obtention d'un consensus. II est à noter que bien que les participants aient été sélectionnés spécifiquement sur la base de leurs antécédents de victimisation par VRA physique, 13 (7,1%) rapportent n’avoir vécu aucune expérience amoureuse difficile notable.

\section{Des expériences amoureuses marquées par la violence subie et perpétrée}

Environ un jeune sur dix ( $n=19,10,3 \%$ ) rapporte un événement de VRA, qu'ils ont subi ou qu'ils ont commis, comme expérience amoureuse la plus difficile qu'ils ont vécu.

Des jeunes qui dévoilent avoir subi des violences dans leurs relations amoureuses. Tout d'abord, bien qu'ils aient tous subi de la VRA physique, nous constatons que peu de garçons qualifient cette expérience comme étant la plus difficile. En effet, des 184 participants, seuls 15 d'entre eux font mention d'un événement relatif à la VRA vécue. De ceux-ci, trois font état de situations de violence menaçant leur intégrité physique, incluant des gestes tels qu'être frappé, giflé, ou retenu de force. Sept participants décrivent différentes formes de violences psychologiques où ils se sont sentis dénigrés, humiliés ou manipulés comme leur pire expérience amoureuse. Trois des participants rapportent des comportements de contrôle, où leurs faits et gestes étaient constamment épiés. Enfin, deux garçons indiquent avoir subi des violences à caractère sexuel de la part de leur partenaire. L'un relate avoir subi des gestes sexuels après avoir refusé des contacts sexuels et l'autre, des blessures génitales.

Des jeunes qui dévoilent avoir commis des gestes violents. Quatre des 184 adolescents révèlent avoir vécu des situations amoureuses complexes, dans lesquelles ils ont eux-mêmes eu recours à des gestes de violence. Entre autres, trois participants relatent des situations d'escalade de violence. Les adolescents font part de réactions agressives des deux partenaires suite à des disputes où le ton monte rapidement. De même, un participant indique que l'expérience amoureuse la plus difficile qu'il ait traversée soit le fait d'avoir posé des gestes d'agression sexuelle.

\section{Des expériences amoureuses marquées par des épreuves à surmonter en couple}

Malgré leurs expériences de victimisation, la majorité des garçons semblent plutôt préoccupés par d'autres blessures amoureuses. Environ un jeune sur cinq $(n=39,21,2 \%)$ mentionne avoir traversé différents obstacles dans leurs relations amoureuses, dont des situations émotionnellement bouleversantes (ex.: des dévoilements d'abus passés), des facteurs externes à la volonté des membres du couple (ex.: déménagement ou désapprobation par l'entourage), ainsi que des disputes.

Des relations marquées de chocs émotionnels. Quelques participants rapportent avoir été confrontés à des situations lors desquelles ils se sont sentis troublés par leur partenaire. Par exemple, trois soulignent avoir reçu des confidences troublantes de la part de leur partenaire, principalement concernant des traumatismes et des agressions sexuelles vécus dans le passé. Trois autres admettent s'être sentis alarmés et inquiets de l'état de santé physique et mentale de leur partenaire concernant, entre autres, des idées dépressives et suicidaires.

Des relations amoureuses compromises par des facteurs extérieurs à la relation. Questionnés sur leur pire expérience amoureuse, 13 des 184 adolescents signalent que leur relation aurait été compromise par des éléments hors de leur contrôle. Huit participants racontent avoir eu à suivre leurs parents lors de déménagements ou de vacances en famille et avoir souffert de cette séparation physique imposée de leur partenaire. D'autres relatent des situations où leurs pairs auraient tenté de diviser leur couple. Bien que ces événements aient été difficiles, selon leurs témoignages, ils auraient aussi permis de mettre à l'épreuve la solidité de leur relation.

Des relations amoureuses marquées de conflits et mésententes. Le thème des conflits est récurrent dans les témoignages recueillis, que 20 participants qualifient comme leur expérience la plus difficile. Ainsi, 14 garçons rapportent avoir vécu des conflits ou des désaccords dans leurs relations amoureuses et avoir trouvé ces tensions comme étant des moments difficiles à traverser. Certains indiquent avoir utilisé de façon constructive ces mésententes au sein de leurs relations amoureuses afin de trouver des solutions à deux. Six autres participants blâment leurs partenaires pour les conflits. 
Tableau 2. Répartition des réponses portant sur la pire expérience vécue dans le cadre d'une relation amoureuse dans la grille de codification ( $n=184$ )

\begin{tabular}{|c|c|c|c|c|}
\hline Catégories & Sous-catégories & $n$ & $\%$ & Verbatims \\
\hline \multirow{8}{*}{$\begin{array}{l}\text { 1. Violence subie } \\
\text { et perpétrée } \\
(n=19 ; 10,3 \%)\end{array}$} & 1.1 Violences subies & 15 & 8,2 & \\
\hline & $\begin{array}{l}\text { 1.1.1 Violences qui atteignent } \\
\text { l'intégrité physique }\end{array}$ & 3 & & $\begin{array}{l}\text { «Après et pendant la chicane, j'ai voulu m'en aller prendre de l'air, fumer une cigarette pour faire tomber la } \\
\text { tension, mais il me retenait violemment pour pas que je parte. » (15 ans) } \\
\text { «De me faire frapper. »(16 ans) }\end{array}$ \\
\hline & $\begin{array}{l}\text { 1.1.2 Dénigrement, humiliation et } \\
\text { manipulation }\end{array}$ & 7 & & $\begin{array}{l}\text { "Ça a duré près d'un an et demi, ma blonde me rabaissait tout le temps en disant que je ne faisais rien pour elle } \\
\text { quand tout le monde me disait que j'étais son esclave. Bref, j'étais de la merde, mais je n'étais pas capable de la } \\
\text { laisser. » (16 ans) } \\
\text { «Des fois, ma blonde est fâchée pour rien, n'importe quand et elle me fait sentir mal, car je l'aime et là, elle va se } \\
\text { confier pas à moi tout de suite pour régler le problème, elle reste là, dit rien et ne me regarde pas pendant des } \\
\text { heures parfois. » (16 ans) }\end{array}$ \\
\hline & 1.1.3 Contrôler les faits et gestes & 3 & & $\begin{array}{l}\text { "Quand ma blonde est dépendante affective et super jalouse ce qui m'empêche de faire le moindre geste sans } \\
\text { me faire envoyer chier. » (17 ans) }\end{array}$ \\
\hline & 1.1.4 Violences liées à la sexualité & 2 & & $\begin{array}{l}\text { "J'étais seul dans la chambre et elle a commencé à me toucher et je ne voulais pas. Elle a continué comme si } \\
\text { j'étais juste un objet sexuel. J'ai dit NON! Elle m'a violé. » (17 ans) } \\
\text { "Après une pénétration du type hardcore, ma blonde en voulait plus mais mon pénis ne fournissait plus, alors } \\
\text { elle s'est mise à le frapper et le mordre. » (17 ans) }\end{array}$ \\
\hline & 1.2 Violences commises & 4 & 2,2 & \\
\hline & 1.2.1 Escalade de la violence & 3 & & $\begin{array}{l}\text { «J'ai surpris une précédente partenaire en pleine relation sexuelle avec un autre homme. J'étais en colère et ma } \\
\text { réaction fut violente envers cet homme. » (17 ans) } \\
\text { « J'étais saoul, elle n'arrêtait pas de crier mon nom et j'ai été près d'elle pour lui crier que je ne suis pas son chien » } \\
\text { (17 ans) }\end{array}$ \\
\hline & $\begin{array}{l}\text { 1.2.2 Violence interpersonnelle } \\
\text { perpétrée }\end{array}$ & 1 & & $\begin{array}{l}\text { "Je me souviens [que] j'étais sur le lit avec [elle] et moi je voulais avoir une relation sexuelle. Elle ne voulait pas } \\
\text { alors je l'ai forcée puis après je me suis senti mal pour elle, j'avais pas respecté son choix. » (16 ans) }\end{array}$ \\
\hline \multirow{6}{*}{$\begin{array}{l}\text { 2. Épreuves à surmonter } \\
\text { en couple } \\
(n=39 ; 21,2 \%)\end{array}$} & 2.1 Chocs émotionnels & 6 & 3,3 & \\
\hline & $\begin{array}{l}\text { 2.1.1 Histoires bouleversantes dans } \\
\text { le passé des membres du couple }\end{array}$ & 3 & & $\begin{array}{l}\text { «Quand ma partenaire m'a avoué avant notre relation amoureuse qu'elle avait été abusée par son oncle dans sa } \\
\text { jeunesse. » (16 ans) }\end{array}$ \\
\hline & $\begin{array}{l}\text { 2.1.2 Être inquiet pour la personne } \\
\text { aimée }\end{array}$ & 3 & & $\begin{array}{l}\text { «Mon ex a eu une dépression qui a duré 3-4 mois, elle s'est coupée et a tenté de se suicider à plusieurs reprises. » } \\
\text { (16 ans) }\end{array}$ \\
\hline & $\begin{array}{l}2.2 \text { Relations amoureuses } \\
\text { compromises par des facteurs } \\
\text { extérieurs au couple }\end{array}$ & 13 & 7,1 & \\
\hline & $\begin{array}{l}\text { 2.2.1 Séparés physiquement malgré } \\
\text { eux }\end{array}$ & 8 & & $\begin{array}{l}\text { «Le fait de savoir qu'elle pourrait déménager bientôt. » ( } 14 \text { ans) } \\
\text { «Lorsque ma blonde est partie en voyage, les premières semaines étaient pas pire mais elle a commencé à } \\
\text { vraiment me manquer. » (16 ans) }\end{array}$ \\
\hline & $\begin{array}{l}\text { 2.2.2 Relations amoureuses } \\
\text { contestées par les pairs }\end{array}$ & 5 & & $\begin{array}{l}\text { «À l'école tout le monde voulait qu'on se sépare alors c'était une situation de confiance entre moi et ma blonde. } \\
\text { Plein de personnes disent des trucs pis on a survécu malgré tout ça. » (16 ans) } \\
\text { « Un autre gars qui veut faire de la marde dans notre couple. » (17 ans) }\end{array}$ \\
\hline
\end{tabular}


Tableau 2. Répartition des réponses portant sur la pire expérience vécue dans le cadre d'une relation amoureuse dans la grille de codification ( $n=184$ ) (suite)

\begin{tabular}{|c|c|c|c|c|}
\hline Catégories & Sous-catégories & $n$ & $\%$ & Verbatims \\
\hline \multirow{3}{*}{$\begin{array}{l}\text { 2. Épreuves à surmonter } \\
\text { en couple } \\
\text { ( } n=39 ; 21,2 \%) \\
\text { (suite) }\end{array}$} & 2.3 Conflits et mésententes & 20 & 10,9 & \\
\hline & 2.3.1 Simples désaccords & 14 & & $\begin{array}{l}\text { "Quand on se chicane c'est les pires moments » (15 ans) } \\
\text { " À cause d'une chicane vraiment niaiseuse, moi et ma blonde ne nous sommes pas vus durant un laps de } \\
\text { temps, mais nous avons finalement réglé nos problèmes en discutant calmement puis tout s'est arrangé. » (15 } \\
\text { ans) }\end{array}$ \\
\hline & 2.3.2 Rejeter la faute sur l'autre & 6 & & $\begin{array}{l}\text { "Une fille jamais satisfaite de ce que l'on fait. » (15 ans) } \\
\text { «ll est facile de devenir impatient avec quelqu'un qui n'est déjà pas patient, moi et ma blonde [on] se chicane } \\
\text { souvent justement à cause de cela. » (17 ans) }\end{array}$ \\
\hline \multirow{6}{*}{$\begin{array}{l}\text { 3. Ruptures amoureuses } \\
(n=47 ; 25,5 \%)\end{array}$} & 3.1 Sentiments de rejet & 25 & 13,6 & \\
\hline & 3.1.1 Le vécu de rupture & 15 & & $\begin{array}{l}\text { «Quand on s'est laissés. » (13 ans) } \\
\text { «C'est quand l'on devait rompre. Je ne savais pas quoi faire j'étais confus et je ne savais pas ce que je voulais. » } \\
\text { (17 ans) }\end{array}$ \\
\hline & 3.1.2 Se sentir utilisé pour le sexe & 2 & & $\begin{array}{l}\text { "Une fille m'a laissé après m'avoir touché plusieurs fois et sucé quelques fois et ça m'a fait mal. Aujourd'hui } \\
\text { encore je crois qu'elle m'aimait que pour ma queue.» (15 ans) }\end{array}$ \\
\hline & $\begin{array}{l}\text { 3.1.3 Rancœur et sensation } \\
\text { d'injustice }\end{array}$ & 8 & & $\begin{array}{l}\text { «Un gars essayait de toucher ma blonde sexuellement, elle était saoule et lui totalement à jeun. Je l'ai frappé à } \\
\text { plusieurs reprises pour ce qu'il avait fait et ma blonde m'a laissé sous prétexte que j'étais trop violent. Elle est allée } \\
\text { voir ce gars-là et ils ont fini en couple. » (15 ans) } \\
\text { "Quand elle m'a laissé pour aucune raison et je l'aimais vraiment. » (16 ans) }\end{array}$ \\
\hline & $\begin{array}{l}3.2 \text { Humeur négative ou } \\
\text { dépressive }\end{array}$ & 13 & 7,1 & $\begin{array}{l}\text { "Quand elle te laisse et que tu penses que c'est fini, je me sentais tellement mal que j'ai été souvent vomir car } \\
\text { j'avais trop de stress. » ( } 16 \text { ans) } \\
\text { "Ma séparation avec mon ex. Je me suis senti anéanti sur tous les niveaux [et j'ai] presque fait une dépression. Je } \\
\text { l'aime toujours et je m'efforce de garder la tête haute et de foncer. » (16 ans) }\end{array}$ \\
\hline & $\begin{array}{l}\text { 3.3 Remords de ne pas avoir de } \\
\text { sentiments amoureux }\end{array}$ & 9 & 4,9 & $\begin{array}{l}\text { «Une fille m'a dit qu'elle m'aimait et qu'elle voulait sortir avec moi. Sans y penser, j'ai accepté, mais je ne l'ai } \\
\text { jamais aimé alors j'ai fait semblant et elle s'en est rendu compte. J'ai cassé et je me sens très mal pour cette } \\
\text { personne. Je lui ai fait beaucoup de peine. » ( } 14 \text { ans) } \\
\text { "Présentement il se passe un froid entre nous deux. Je ressens moins d'amour pour elle que j'en éprouvais } \\
\text { auparavant. Elle tente constamment de me convaincre que ça marche encore, mais à mon avis c'est terminé. » } \\
(15 \text { ans) }\end{array}$ \\
\hline \multirow{5}{*}{$\begin{array}{l}\text { 4. Enjeux d'engagement, } \\
\text { instabilité dans la } \\
\text { relation et infidélité } \\
(n=57 ; 31 \%)\end{array}$} & 4.1 Méfiance l'un envers l'autre & 12 & 6,5 & \\
\hline & $\begin{array}{l}\text { 4.1.1 Dynamiques empreintes de } \\
\text { jalousie }\end{array}$ & 9 & & $\begin{array}{l}\text { "La période la plus dure est quand mon ex était devenue jalouse, je n'étais plus capable. » (16 ans) } \\
\text { "Ma blonde est souvent jalouse si je vais voir une amie et moi aussi je suis jaloux quand elle va voir un autre } \\
\text { gars. » (18 ans) }\end{array}$ \\
\hline & 4.1.2 Abus de confiance & 3 & & $\begin{array}{l}\text { «J'ai eu de la misère à accepter le fait qu'elle parlait dans mon dos et disait des conneries à mon égard, j'ai été } \\
\text { déçu de voir comment elle pouvait agir. » (16 ans) }\end{array}$ \\
\hline & $\begin{array}{l}4.2 \text { Instabilité dans le niveau } \\
\text { d'engagement amoureux }\end{array}$ & 25 & 13,5 & \\
\hline & 4.2.1 Triangles amoureux & 8 & & $\begin{array}{l}\text { "Le moment où nous nous sommes laissé quelque temps (nous sommes revenus ensemble par la suite) et } \\
\text { qu'elle voyait un garçon en particulier. Ça m'a fait très mal et même encore aujourd'hui, même si cela date de } \\
\text { longtemps, ça me touche toujours. » (15 ans) } \\
\text { "Quand j'ai eu peur de perdre ma blonde à cause de son ex. » (16 ans) }\end{array}$ \\
\hline
\end{tabular}


Expérience amoureuse la plus difficile :

Qu'en disent les garçons rapportant un vécu de violence physique dans leurs relations amoureuses?

Tableau 2. Répartition des réponses portant sur la pire expérience vécue dans le cadre d'une relation amoureuse dans la grille de codification ( $n=184$ ) (suite)

\begin{tabular}{|c|c|c|c|c|}
\hline Catégories & Sous-catégories & $n$ & $\%$ & Verbatims \\
\hline \multirow{7}{*}{$\begin{array}{l}\text { 4. Enjeux d'engagement, } \\
\text { instabilité dans la } \\
\text { relation et infidélité } \\
(n=57 ; 31 \%) \\
\text { (suite) }\end{array}$} & $\begin{array}{l}\text { 4.2.2 Relations amoureuses qui } \\
\text { vacillent puis reprennent }\end{array}$ & 11 & & $\begin{array}{l}\text { «En ce moment on vient de se remettre d'une pause alors je suis plutôt joyeux sinon l'expérience la plus difficile } \\
\text { c'était cette pause. » (15 ans) } \\
\text { «Quand nous nous sommes laissés à plusieurs reprises. » (17 ans) }\end{array}$ \\
\hline & 4.2.3 Instabilité du début de relation & 4 & & « Faire en sorte que notre relation fonctionne à long terme avec elle. » (15 ans) \\
\hline & $\begin{array}{l}\text { 4.2.4 Préférer la liberté à } \\
\text { l'engagement }\end{array}$ & 2 & & $\begin{array}{l}\text { «L'amour c'est de la marde. Dans [la] vie, [i]] faut avoir du fun sortir jouir et baiser. Merci. » } \\
\text { «An argument over commitment issues. » }\end{array}$ \\
\hline & $\begin{array}{l}4.3 \text { Relations amoureuses } \\
\text { marquées par l'infidélité }\end{array}$ & 13 & 7,1 & \\
\hline & 4.3.1 Le vécu d'infidélité & 10 & & $\begin{array}{l}\text { «L'expérience la plus difficile est de m'avoir fait tromper par la fille que j'aime dans un party. » ( } 16 \text { ans) } \\
\text { "Je me suis déjà fait tromper par ma blonde et je ne me sentais plus important pour elle, je croyais qu'elle ne } \\
\text { m'aimait plus et je n'avais plus confiance en elle. » (17 ans) }\end{array}$ \\
\hline & $\begin{array}{l}4.3 .2 \text { Craindre une infidélité } \\
\text { imminente }\end{array}$ & 3 & & « Lorsque ta blonde fréquente d'autres garçons et que tu penses qu'elle va te tromper.» (16 ans) \\
\hline & $\begin{array}{l}\text { 4.4 Remords à l'idée de trahir et } \\
\text { d'être infidèle }\end{array}$ & 7 & 3,8 & $\begin{array}{l}\text { "Je l'ai trahie en disant quelque chose que je n'aurais pas dû à mon meilleur ami, en rapport avec mes relations } \\
\text { sexuelles. » (15 ans) } \\
\text { "J'étais à un party et j'ai été voir ailleurs. Sur le moment, je me foutais des conséquences. Mais par la suite les } \\
\text { remords sont arrivés. » (âge non inscrit) }\end{array}$ \\
\hline \multirow{5}{*}{$\begin{array}{l}\text { 5. Besoins sexuels non } \\
\text { concordants, expériences } \\
\text { inconfortables, et } \\
\text { accidents contraceptifs } \\
(n=9 ; 4,9 \%)\end{array}$} & $\begin{array}{l}5.1 \text { Inconfort dans leur } \\
\text { découverte de la sexualité }\end{array}$ & 5 & 2,7 & \\
\hline & $\begin{array}{l}\text { 5.1.1 Premières relations sexuelles } \\
\text { marquées par des douleurs }\end{array}$ & 2 & & $\begin{array}{l}\text { "Les premières relations sexuelles avec ma blonde ont été plutôt difficiles, aucun de nous deux n'a joui avant } \\
\text { cinq ou six essais ou relations. Elle à cause de la douleur et moi à cause que je me sentais mal pour elle.» ( } 16 \text { ans) }\end{array}$ \\
\hline & $\begin{array}{l}\text { 5.1.2 Relations sexuelles marquées } \\
\text { par des besoins sexuels non } \\
\text { concordants }\end{array}$ & & & $\begin{array}{l}\text { "Cette année, avec une fille hyper pas déniaisée et ça me tue, mais il [n']y aura jamais de violence. Si c'est le cas, } \\
\text { j'ai qu'à finir la relation et ça sera fini. » (14 ans) }\end{array}$ \\
\hline & $\begin{array}{l}5.2 \text { Contextes qui entravent } \\
\text { l'intimité sexuelle }\end{array}$ & 2 & 1,1 & « Nous allions avoir une relation sexuelle et sa mère est arrivée. » (16 ans) \\
\hline & 5.3 Des enjeux contraceptifs & 2 & 1,1 & $\begin{array}{l}\text { "When I was having sex with my ex-girlfriend a month after we broke up and the condom broke. We had to get } \\
\text { a plan B pill and it was very stressfull. Me and my girlfriend broke up cause I can't handle a relationship. » (16 ans) }\end{array}$ \\
\hline
\end{tabular}




\section{Les ruptures amoureuses}

Un autre thème fréquemment rapporté comme étant le plus difficile est celui des ruptures. Environ le quart des participants ( $n=47,25,5 \%$ ) rapporte que la fin d'une relation est l'expérience amoureuse la plus difficile qu'ils ont eu à surmonter. Certains expriment s'être sentis accablés par un sentiment de rejet, la fin imminente de leur relation ou encore avoir été rongés par la culpabilité d'avoir quitté leur partenaire.

Des sentiments de rejet. Parmi les 25 témoignages figurant dans cette catégorie, les participants indiquent s'être sentis blessés par la séparation et mentionnent avoir eu l'impression d'être utilisés ou trahis au moment de la rupture. Plus précisément, 15 garçons décrivent s'être sentis abandonnés ou confus durant cette épreuve. Deux d'entre eux révèlent qu'ils ont eu l'impression d'avoir été utilisés à des fins sexuelles par leur ancien.ne partenaire pour ensuite être «jetés ». Aussi, une amertume se dégage des propos recueillis, certains participants qualifiant la rupture comme une injustice.

Une humeur négative ou dépressive. Dans le même ordre d'idées, 13 participants tiennent des propos sombres en décrivant la fin de leur relation amoureuse. Ils racontent comment ces événements ont entraîné certains symptômes physiques et psychologiques, comme des nausées et un abattement émotionnel.

Des remords à l'idée de ne pas avoir de sentiments amoureux. Neuf participants indiquent vivre de la culpabilité de ne plus se sentir amoureux de leur partenaire et de devoir mettre un terme à la relation comme étant l'expérience la plus difficile vécue dans leur vie amoureuse. Ils déplorent particulièrement d'avoir fait de la peine à l'autre.

\section{Des expériences amoureuses marquées par des enjeux d'engagement, d'instabilité et d'infidélité}

II s'agit de la catégorie regroupant le plus de participants. Près du tiers ( $n=57,31 \%)$ signale avoir trouvé ardu de traverser des situations d'infidélité ou de trahison. Ils indiquent s'être sentis méfiants envers leur partenaire, avoir ressenti un désir d'engagement divergent de celui de leur partenaire, s'être sentis jaloux ou regretter d'avoir euxmêmes posé des gestes d'infidélité.

Des jeunes qui vivent dans la méfiance l'un envers l'autre. Les pires expériences amoureuses sont qualifiées comme empreintes de méfiance pour neuf participants qui indiquent avoir vécu des dynamiques marquées par la jalousie. Ce serait parfois le partenaire, parfois le participant, ou encore les deux membres simultanément, qui auraient vécu cette jalousie. Trois autres garçons mentionnent s'être sentis trahis par leur partenaire qui aurait abusé de leur confiance en médisant à leur sujet.

Un niveau d'engagement amoureux instable. Huit garçons rapportent avoir fait partie d'un triangle amoureux. Onze jeunes signalent des situations où ils auraient rompu à plusieurs reprises, puis se seraient réconciliés. Quatre participants nomment que l'instabilité présente en début de relation et leur désir de faire durer la relation avait été l'obstacle le plus difficile qu'ils ont vécu en contexte amoureux. Deux autres racontent avoir eu de la difficulté à être pleinement engagés dans une relation amoureuse.

Des relations amoureuses marquées par l'infidélité. Une expérience d'infidélité et le sentiment d'abandon associé sont énoncés par une dizaine de participants comme l'expérience la plus difficile qu'ils aient vécue en relation amoureuse. Ils craignent que leur confiance en eux et que leur capacité à faire confiance à des partenaires dans l'avenir soit ébranlée. De même, trois adolescents font mention de craindre que leur partenaire pose imminemment un geste d'infidélité.

Des remords à l'idée de trahir et d'être infidèle. Des propos liés à de lourds sentiments de culpabilité ont été énoncés par sept garçons. Ils indiquent avoir eux-mêmes trompé la confiance de leur partenaire, avoir commis des gestes d'infidélité ou encore avoir révélé des confidences.

5. Une sexualité marquée par des besoins non concordants, des expériences inconfortables, et des accidents contraceptifs

Neuf des 184 participants (4,9\%) révèlent avoir vécu des événements difficiles à travers leurs expériences sexuelles. Ces témoignages portent sur des interactions sexuelles inconfortables, des difficultés à se retrouver en toute intimité, ainsi que des accidents contraceptifs.

Des jeunes qui vivent un inconfort dans leur découverte de la sexualité. Deux participants rapportent que 
leurs premiers rapports sexuels auraient été insatisfaisants. Ils mentionnent des difficultés à atteindre l'orgasme, ainsi que des douleurs vaginales chez leur partenaire. Trois autres indiquent que leur niveau de désir sexuel ne convergeait pas avec celui de leur partenaire.

Des contextes qui entravent l'intimité sexuelle. De même, deux garçons révèlent des situations ne permettant pas d'approfondir la découverte de leur sexualité par manque d'opportunité pour avoir des moments intimes. Par exemple, certains auraient été surpris par leurs parents lors de relations sexuelles.

Des enjeux contraceptifs. Enfin, deux participants rapportent des accidents contraceptifs (par ex. : rupture de préservatif) et avoir craint une grossesse comme leur expérience amoureuse la plus difficile.

\section{Approfondissement des résultats par des analyses quantitatives}

Afin de documenter chez les participants les facteurs de risque associés aux expériences de VRA physique, des analyses statistiques corrélationnelles et de comparaison de moyennes ont été menées. En premier lieu, des analyses menées auprès des 1701 garçons ayant participé à l'enquête PAJ et ayant été en relation amoureuse dans les 12 derniers mois ont permis de comparer les résultats obtenus aux échelles APNS, CADRI et SES entre ceux qui rapportent de la VRA physique durant cette période et ceux n'en rapportant pas. Ainsi, les résultats du Test $t$ soulignent que les garçons ayant été victimes de VRA physique se montrent significativement plus tolérants envers la VRA physique $(M=4,43, E . T .=3,57)$ que ceux n'ayant pas vécu ce type de violence $(M=3,05, E . T .=3,19 ; t(304,74)=-5,64, p=, 001$, I.C. $95 \%=-1,87$ à -,90). En second lieu, des corrélations de Pearson effectuées parmi les 184 participants mettent en lumière que plus un participant rapporte une tolérance élevée envers la violence, plus il a vécu de formes différentes de VRA $(r=, 20, p=, 035$, I.C. $95 \%=, 02$ à ,37).

\section{Discussion}

Les données empiriques et les mouvements sociaux visant à reconnaître la victimisation par VRA physique des garçons adolescents sont de plus en plus présents. II apparait fondamental de donner la parole à ces jeunes et de documenter leurs expériences. Ainsi, dans cette analyse menée auprès d'adolescents ayant été victimes de VRA physique, les témoignages révèlent que la majorité des participants ne décrivent pas les événements de violence comme l'expérience amoureuse la plus difficile qu'ils aient vécue. Malgré tout, ils soulignent la présence de plusieurs sources de détresse dans leurs relations amoureuses, qui semblent se cumuler à leurs expériences de victimisation et pourraient ainsi limiter leur capacité à avoir accès à leurs ressources en matière de résilience et à solliciter de l'aide. Certains extraits de discours suggèrent la présence de dynamiques amoureuses problématiques et de gestes réciproques de VRA.

\section{Tolérer la VRA et les dynamiques relationnelles problématiques}

Les conflits, les ruptures et les infidélités représentent les thèmes les plus fréquemment rapportés par les participants en tant que pire expérience amoureuse. Ces résultats sont cohérents avec les connaissances actuelles, ces enjeux pouvant être le signe de dynamiques amoureuses problématiques au même titre que la VRA (Paat et Markham, 2019). Il est effectivement reconnu que les ruptures peuvent être plus souffrantes que la VRA, la violence étant parfois perçue comme une démonstration d'attention ou une façon de demander de l'attention, voire une preuve d'amour, particulièrement chez les jeunes qui ont grandi dans un environnement familial empreint de violence (Pepler, 2012). Selon les études, la difficulté à exprimer ses ressentis et ses besoins serait associée à des dynamiques de couple néfastes et empreintes de violence (Gallaty et Zimmer-Gembeck, 2008; Paat et Markham, 2019). Ces dynamiques, perceptions et difficultés pourraient aussi être liées à une plus grande tolérance envers les comportements violents, de même qu'à une difficulté à se reconnaître comme victime et chercher de l'aide (Ali et al., 2011; García-Díaz et al., 2017; Mahalik et al., 2010; Martin et al., 2012).

Les analyses statistiques soulignent que plus un garçon endosse des attitudes favorisant la VRA physique, plus le risque de subir de la VRA physique serait élevé. De même, le fait d'avoir vécu plusieurs formes de violence (physique, psychologique et sexuelle) et de dynamiques relationnelles malsaines est associé à une plus grande tolérance envers la VRA. Ces résultats sont cohérents avec les conclusions de travaux antérieurs soulignant qu'une grande tolérance envers la violence, que ce soit dans un contexte amoureux ou non, est liée chez les garçons au risque de subir de la VRA physique (Ali et al., 2011; Reyes et al., 2016). De même, percevoir la violence comme ayant un rôle positif dans une relation amoureuse est lié à des attitudes tolérantes envers la VRA (García-Díaz et al., 2017). 


\section{Se reconnaître comme ayant été victime}

Certains discours sociaux peuvent affecter la capacité des garçons à reconnaître les gestes violents qu'ils subissent ou les dynamiques amoureuses problématiques dans lesquelles ils évoluent. Reyes et ses collaborateurs (2016) soutiennent que l'idée que tolérer la VRA pourrait concorder avec certaines normes de genre stéréotypées. Par exemple, plusieurs scripts socioculturels prescrivent aux garçons et aux hommes de se montrer agressifs, dominants et émotionnellement détachés (Easton et al., 2014; Eaton et Rose, 2011; Mahalik et al., 2010; Oransky et Marecek, 2009). De plus, certaines normes incitent à tolérer davantage la violence physique lorsqu'elle ne mène pas à des blessures sévères, ou lorsqu'elle est perpétrée par une fille plutôt que par un garçon (Muñoz-Rivas et al., 2021; Ruel et al., 2020; Simon et al., 2010). Dans ce contexte, les garçons qui sont victimes de VRA de la part d'une fille pourraient ne pas se sentir légitimes de la dénoncer ou ne pas être pris au sérieux lorsqu'ils la dénoncent. Ainsi, il peut être difficile pour les garçons de reconnaitre des dynamiques amoureuses problématiques, de la VRA subie ou de s'identifier comme victimes de VRA, même s'ils connaissent les comportements de violence en contexte de relation amoureuse, comme pousser, frapper, gifler ou brutaliser le partenaire.

Ces normes peuvent donc faire obstacle à la capacité des garçons à reconnaître ces expériences de VRA comme source de détresse, comme si elles constituaient une faille à leur masculinité ou une preuve de vulnérabilité (Martin et al., 2012; Easton et al., 2014). De plus, des études démontrent que l'adhésion aux stéréotypes de masculinité est liée à davantage de détresse psychologique et fait entrave à la capacité à solliciter de l'aide (Easton et al., 2014; Mahalik et al., 2010; Martin et al., 2012). Bien qu'être en mesure d'identifier leur détresse et les comportements violents et problématiques de leurs partenaires agisse comme source de résilience, les études soulignent que les garçons sont moins enclins que les filles à solliciter du soutien en contexte de VRA (Easton et al., 2014; Martin et al., 2012). II est documenté que non seulement les adolescents, mais également les hommes adultes tendent également à trouver difficile de se reconnaître comme victime, à chercher de l'aide, et à sentir qu'ils sont pris au sérieux lorsqu'ils subissent de la violence conjugale (Mahalik et al., 2010; Vasseur et al., 2020). Cela suggère que l'adhésion aux normes et stéréotypes de genre peut engendrer des obstacles à la recherche d'aide se poursuivant jusque dans l'âge adulte.

\section{Limites de cette recherche et futures études}

La présente étude innove en proposant une exploration du vécu de VRA physique chez les garçons adolescents en ayant recours à la fois à des analyses qualitatives et quantitatives. Toutefois, certaines limites doivent être soulignées. Mentionnons que les témoignages ont été obtenus par écrit à l'aide d'une question ouverte, et non par entrevue approfondie. Ainsi, quelques-unes des réponses analysées sont brèves et les éléments contextuels sont peu détaillés. De plus, la formulation de cette question ne portait pas directement sur la VRA, et ne demandait pas spécifiquement de se référer à la relation ou à la période dans laquelle la VRA a été subie, ce qui a pu orienter différemment les réponses. Bien que la mesure utilisée permette de dépister et documenter les comportements de VRA physique vécus, elle ne permet pas de les détailler, d'en décrire le contexte ou les motivations, ni d'approfondir les stratégies de recherche d'aide mises en place par les garçons suivant ces expériences.

Nous constatons que certains participants relatent des événements où ils auraient eux aussi exercé de la violence envers leur partenaire, ce qui suggère la présence dans certains cas de violence bidirectionnelle et des dynamiques d'escalade de violence. Ce constat témoigne de la pertinence de continuer d'étudier les contextes dans lesquels les garçons vivent des expériences de VRA afin de mieux comprendre les dynamiques de violence, le vécu des garçons dans ces situations et les facteurs de risque et de protection associés. Des méthodes qualitatives permettant un plus grand approfondissement du discours et du vécu de VRA des garçons adolescents, tel que le recours à des entrevues approfondies, seraient à privilégier. Les études à venir devraient également se pencher sur les spécificités dans le vécu de VRA physique selon l'orientation sexuelle des participants, afin de nous assurer de développer des interventions adaptées à la diversité des besoins et des réalités relationnelles et sexuelles. Il serait aussi intéressant pour de futures études de répéter les analyses statistiques en utilisant des variables de contrôle, comme avoir subi ou avoir été témoin d'événements de violence durant l'enfance. 


\section{Implications pratiques}

Ces données font état des préoccupations amoureuses de garçons ayant subi de la VRA physique et appuient la pertinence de mettre en place des interventions éducatives et préventives à propos des relations amoureuses et des dynamiques saines et malsaines. Comme le relèvent les résultats de la présente étude, les thèmes clés à aborder dans ces interventions sont les enjeux liés à la rupture, à l'infidélité, aux conflits, à l'instabilité relationnelle, aux difficultés sexuelles et à la violence.

Les interventions gagneraient à aider les garçons à développer leurs capacités de résilience, entre autres, en les sensibilisant au fait que les garçons peuvent également être victimes de VRA physique, et en les soutenant dans l'identification des différentes formes de VRA, l'identification de leur détresse et le développement de leurs capacités à dévoiler et à chercher du soutien auprès de ressources appropriées (Grych et al., 2015; Helm et al., 2017). En effet, des travaux soulèvent que les garçons et les hommes sont plus réticents à dévoiler des abus subis, mais qu'ils y parviennent plus facilement s'ils sont accompagnés et soutenus dans leurs démarches (Easton, 2013; Martin et al., 2012).

II semble aussi essentiel d'œuvrer à mettre en place des interventions d'éducation à la sexualité et de prévention de la violence visant à assouplir l'adhésion aux stéréotypes socioculturels de masculinité et à diminuer les attitudes tolérantes envers la violence. Ces initiatives pourraient passer par la promotion de relations amoureuses saines et égalitaires et la reconnaissance que la VRA est inacceptable, peu importe sa sévérité, de façon à encourager les garçons victimes à solliciter de l'aide. Considérant le stoïcisme et la continence émotionnelle que les normes socioculturelles peuvent imposer aux garçons, l'expression saine de leurs ressentis devrait aussi être au centre des interventions éducatives réalisées afin de favoriser la résilience (Easton et al., 2014; Eaton et Rose, 2011; Kassis et al., 2013).

Finalement, notons qu'il reste un vaste travail à faire en ce qui concerne l'accès aux ressources spécialisées pour les garçons (et les hommes) victimes de VRA, d'où l'importance d'intervenir en amont en conduisant des campagnes de sensibilisation destinées aux jeunes, aux intervenants et aux autres adultes significatifs au sujet des premières relations amoureuses et des défis qui leur sont associés. La spécificité de la victimisation chez les garçons devrait être étudiée plus en profondeur afin de favoriser le soutien et la prévention par les pairs qui peuvent agir comme témoins ou confidents. Les approches ciblant l'entraide par les pairs se sont d'ailleurs révélées être efficaces afin d'améliorer les connaissances au sujet de la VRA, le sentiment d'auto-efficacité, la qualité de la communication et la capacité de résilience (Fernet et al., 2019).

\section{Remerciement}

Les auteurs remercient les adolescents, de même que les écoles et commissions scolaires ayant pris part à l'Enquête Parcours Amoureux des Jeunes (PAJ). Nous tenons également à remercier Catherine Moreau pour la coordination du projet et Manon Robichaud pour la gestion des banques de données.

\section{Financement}

Cette étude est rendue possible grâce au soutien financier de I'Institut de Recherche en Santé du Canada (IRSC; \#103944) accordé à Martine Hébert.

\section{Conflits d'intérêts}

Les auteurs n'ont pas de conflits d'intérêts à déclarer.

\section{References}

Ali, B., Swahn, M. et Hamburger, M. (2011). Attitudes affecting physical dating violence perpetration and victimization: Findings from adolescents in a high-risk urban community. Violence and Victims, 26(5), 669-683. https://doi.org/10.1891/0886-6708.26.5.669

BBC Three. (2017). What happens when a woman abuses a man in public? [vidéo]. https://www.youtube.com/watch?v=GccCWo_eZdw

Braun, V. et Clarke, V. (2006). Using thematic analysis in psychology. Qualitative Research in Psychology, 3(2), 77-101. https://doi.org/10.1191/1478088706qp063oa

Eaton, A. A. et Rose, S. (2011). Has dating become more egalitarian? A 35 years review using Sex Roles. Sex Roles, 64(11-12), 843-862. 
https://doi.org/10.1007/s11199-011-9957-9

Easton, S. D. (2013). Disclosure of child sexual abuse among adult male survivors. Clinical Social Work Journal, 41(4), $344-355$. https://doi.org/10.1007/s10615-012-0420-3

Easton, S. D., Saltzman, L. Y. et Willis, D. G. (2014). Would you tell under circumstances like that?": Barriers to disclosure of child sexual abuse for men. Psychology of Men \& Masculinity, 15(4), 460-469. https://doi.org/10.1037/a0034223

Fernet, M., Hébert, M., Dion, J., Fortin, A., Brodeur, G., Dupuis, C. M. et Asselin, M. D. (2019). Les enjeux de prévention de la violence dans les relations amoureuses chez les jeunes: Une synthèse des programmes ciblant la recherche d'aide et l'entraide par les pairs [Manuscrit sous presse]. Dans Pratiques et recherches féministes en matière de violence conjugale. Co-construction des connaissances et expertises. Presses de l'Université du Québec.

Foshee, V. A., Linder, F., MacDougall, J. E. et Bangdiwala, S. (2001). Gender differences in the longitudinal predictors of adolescent dating violence. Preventive Medicine, 32(2), 128-141. https://doi.org/10.1006/pmed.2000.0793

Foshee, V. A., Reyes, H. L. M., Gottfredson, N. C., Chang, L. Y. et Ennett, S. T. (2013). A longitudinal examination of psychological, behavioral, academic, and relationship consequences of dating abuse victimization among a primarily rural sample of adolescents. Journal of Adolescent Health, 53(6), 723-729. https://doi.org/10.1016/j.jadohealth.2013.06.016

Fourboul, C. V. (2012). Ce que « analyse de données qualitatives » veut dire. Revue Internationale de Psychosociologie, 18(44), 71-88. https://doi.org/10.3917/rips.044.0071

Gallaty, K. et Zimmer-Gembeck, M. J. (2008). The daily social and emotional worlds of adolescents who are psychologically maltreated by their romantic partners. Journal of Youth and Adolescence, 373), 310-323. https:/.doi.org/10.1007/s10964-007-9248-5

García-Díaz, V., Bringas, C., Fernández-Feito, A., Antuña, M. Á., Lana, A., Rodríguez-Franco, L., et Rodríguez-Díaz, F. J. (2017). Tolerance and perception of abuse in youth dating relationships. Journal of Aggression, Maltreatment \& Trauma, 26(5), $462-474$. https://doi.org/10.1080/10926771.2017.1304477

Grych, J., Hamby, S. et Banyard, V. (2015). The resilience portfolio model: Understanding healthy adaptation in victims of violence. Psychology of Violence, 5(4), 343-354. https://doi.org/10.1037/a0039671

Hébert, M., Blais, M. et Lavoie, F. (2011). Enquête sur le Parcours amoureux des jeunes - volet I: Enquête longitudinale représentative auprès des jeunes Québécois fréquentant l'école secondaire. Université du Québec à Montréal.

Hébert, M., Blais, M. et Lavoie, F. (2017). Prevalence of teen dating victimization among a representative sample of high school students in Quebec. International Journal of Clinical and Health Psychology, 17(3), 225-233. https://doi.org/10.1016/j.jichp.2017.06.001

Hébert, M., Lapierre, A., Lavoie, F., Fernet, M. et Blais, M. (2018). La violence dans les relations amoureuses des jeunes. Dans J. Laforest, P. Maurice et L. M. Bouchard (dir.), Rapport québécois sur la violence et la santé (p. 97-129). Institut national de santé publique du Québec. https://www.inspq.qc.ca/rapport-quebecois-sur-la-violence-et-la-sante/la-violence-dans-les-relationsamoureuses-des-jeunes

Helm, S., Baker, C. K., Berlin, J. et Kimura, S. (2017). Getting in, being in, staying in, and getting out: Adolescents' descriptions of dating and dating violence. Youth \& Society, 49(3), 318-340. https://doi.org/10.1177/0044118X15575290

Kassis, W., Artz, S., Scambor, C., Scambor, E. et Moldenhauer, S. (2013). Finding the way out: A non-dichotomous understanding of violence and depression resilience of adolescents who are exposed to family violence. Child Abuse \& Neglect, 37(2-3), 181199. https://doi.org/10.1016/j.chiabu.2012.11.001

Koss, M. P., Abbey, A., Campbell, R., Cook, S., Norris, J., Testa, M., Ullman, S., West, C. et White, J. (2007). Revising the SES: A collaborative process to improve assessment of sexual aggression and victimization. Psychology of Women Quarterly, 31(4), 357-370. https://doi.org/10.1111/j.1471-6402.2008.00468.x

Machado, A., Hines, D. et Matos, M. (2016). Help-seeking and needs of male victims of intimate partner violence in Portugal. Psychology of Men \& Masculinity, 17(3), 255-264. https://doi.org/10.1037/men0000013

Mahalik, J. R., Good, G. E. et Englar-Carlson, M. (2010). Masculinity scripts, presenting concerns, and help seeking: Implications for practice and training. Dans S. R. Harper et F. Harris III (dir.), College men and masculinities: Theory, research, and implications for practice (1ère éd., p. 77-96). Jossey-Bass.

Martin, C. E., Houston, A. M., Mmari, K. N. et Decker, M. R. (2012). Urban teens and young adults describe drama, disrespect, dating violence and help-seeking preferences. Maternal and Child Health Journal, 16(5), 957-966. http://doi.org/10.1007/s10995-0110819-4

Muñoz-Rivas, M. J., Graña, J. L., O'Leary, K. D. et González, M. P. (2007). Aggression in adolescent dating relationships: Prevalence, justification, and health consequences. Journal of Adolescent Health, 40(4), 298-304. https://doi.org/10.1016/j.jadohealth.2006.11.137

Oransky, M. et Marecek, J. (2009). "I'm Not Going to Be a Girl" Masculinity and emotions in boys' friendships and peer groups. Journal of Adolescent Research, 24(2), 218-241. https://doi.org/10.1177/0743558408329951 
Paat, Y. F. et Markham, C. (2019). The roles of family factors and relationship dynamics on dating violence victimization and perpetration among college men and women in emerging adulthood. Journal of Interpersonal Violence, 34(1), 81-114. https://doi.org/10.1177/0886260516640544

Pepler, D. (2012). The development of dating violence: What doesn't develop, what does develop, how does it develop, and what can we do about it? Prevention Science, 13(4), 402-409. https://doi.org/10.1007/s11121-012-0308-z

Poupart, J. (1997). L'entretien de type qualitatif : considérations épistémologiques, théoriques et méthodologiques. Dans J. Poupart, J. P. Deslauriers, L. H. Groulx, A. Laperrière, R. Mayer, A. P. Pires (dir.), La recherche qualitative: Enjeux épistémologiques et méthodologiques (p. 173-210). Gaetan Morin Editeur.

Project Unbreakable. (s.d.). https://projectunbreakable.tumblr.com/post/155746893802/hi-everyone-in-light-of-our-currentpolitical

Reyes, H. L. M., Foshee, V. A., Niolon, P. H., Reidy, D. E. et Hall, J. E. (2016). Gender role attitudes and male adolescent dating violence perpetration: Normative beliefs as moderators. Journal of Youth and Adolescence, 45(2), 350-360. https://doi.org/10.1007/s10964-015-0278-0

Ruel, C., Lavoie, F., Hébert, M. et Blais, M. (2020). Gender's role in exposure to interparental violence, acceptance of violence, selfefficacy, and physical teen dating violence among Quebec adolescents. Journal of Interpersonal Violence, 35(15-16), 30793101. https://doi.org/10.1177/0886260517707311

Simon, T. R., Miller, S., Gorman-Smith, D., Orpinas, P. et Sullivan, T. (2010). Physical dating violence norms and behavior among sixthgrade students from four US sites. The Journal of Early Adolescence, 30(3), $395-409$. https://doi.org/10.1177/0272431609333301

Vagi, K. J., Olsen, E. O. M., Basile, K. C. et Vivolo-Kantor, A. M. (2015). Teen dating violence (physical and sexual) among US high school students: Findings from the 2013 National Youth Risk Behavior Survey. JAMA Pediatrics, 169(5), $474-482$. http://doi.org/10.1001/jamapediatrics.2014.3577

Van Camp, T., Hébert, M., Guidi, E., Lavoie, F. et Blais, M. (2014). Teens' self-efficacy to deal with dating violence as victim, perpetrator or bystander. International Review of Victimology, 20(3), 289-303. https://doi.org/10.1177/0269758014521741

Vasseur, P., Dupont, M. et Rey-Salmon, C. (2020). Hommes victimes de violences conjugales constats et perspectives. La Revue de Médecine Légale, 11(2), 56-62. https://doi.org/10.1016/j.medleg.2020.03.001

Wekerle, C., Leung, E., Wall, A. M., MacMillan, H., Boyle, M., Trocme, N. et Waechter, R. (2009). The contribution of childhood emotional abuse to teen dating violence among child protective services-involved youth. Child Abuse \& Neglect, 33(1), 45-58. https://doi.org/10.1016/j.chiabu.2008.12.006

Wolfe, D. A., Scott, K., Reitzel-Jaffe, D., Wekerle, C., Grasley, C. et Straatman, A. L. (2001). Development and validation of the conflict in adolescent dating relationships inventory. Psychological Assessment, 13(2), 277-293. https://doi.org/10.1037/10403590.13.2.277 